\title{
LIMITS OF PURE STATES
}

\author{
by R. J. ARCHBOLD
}

(Received 25th November 1987)

\section{Introduction}

In [7, Section 5], Glimm showed that if $\phi$ and $\psi$ are inequivalent pure states of a liminal $C^{*}$-algebra $A$ such that the Gelfand-Naimark-Segal (GNS) representations $\pi_{\phi}$ and $\pi_{\psi}$ cannot be separated by disjoint open subsets of the spectrum $\hat{A}$ then $\frac{1}{2}(\phi+\psi)$ is a weak*-limit of pure states. We extend this to arbitrary $C^{*}$-algebras (and more general convex combinations) by means of what we hope will be regarded as a transparent proof based on the notion of transition probabilities. As an application, we show that if $J$ is a proper primal ideal of a separable $C^{*}$-algebra $A$ then there exists a state $\phi$ in $\overline{P(A)}$ (the pure state space) such that $J=\operatorname{ker} \pi_{\phi}$ (Theorem 3 ). The significance of this is discussed below after the introduction of further notation and terminology.

The state space $S(A)$ is defined by

$$
S(A)=\left\{\phi \in A^{*}: \phi \geqq 0,\|\phi\|=1\right\} .
$$

The set $P(A)$ of pure states consists of the extreme points of $S(A)$ and the set $F(A)$ of factorial states consists of those $\phi \in S(A)$ such that the von Neumann algebra generated by $\pi_{\phi}(A)$ is a factor. Unless stated otherwise, $A^{*}$ should be regarded as being endowed with the weak*-topology. The closures $\overline{P(A)}$ and $\overline{F(A)}$ are known respectively as the pure and factorial state spaces of $A$.

Following [2, Definition 3.1], we say that a (closed two-sided) ideal $J$ of $A$ is primal if whenever $n \geqq 2$ and $J_{1}, J_{2}, \ldots, J_{n}$ are ideals of $A$ such that $J_{1} J_{2} \ldots J_{n}=\{0\}$ then $J_{i} \subseteq J$ for at least one value of $i$. It is shown in [2, Theorem 3.5] that if $\phi \in S(A)$ then $\phi \in \overline{F(A)}$ if and only if ker $\pi_{\phi}$ is primal. Thus there is a mapping $\theta$ from $\overline{F(A)} \cap S(A)$ into the set of proper primal ideals of $A$ given by $\theta(\phi)=\operatorname{ker} \pi_{\phi}$ The mapping $\theta$ is continuous and open relative to its image [1, Theorem 3.6], and is surjective if $A$ is separable (see [2, p. 62]). Theorem 3 tells us that, if $A$ is separable, the restriction of $\theta$ to $\overline{P(A)} \cap S(A)$ is still surjective.

We note here two related questions to which we have been able to find affirmative answers only in certain special cases.

Question 1. Does $\theta(\overline{F(A)} \cap S(A))=\theta(\overline{P(A)} \cap S(A))$ for all $C^{*}$-algebras $A$ ? 
Question 2. Is the restriction of $\theta$ to $\overline{P(A)} \cap S(A)$ an open mapping relative to its image?

It is a pleasure to acknowledge that part of this work was carried out at the 1986/7 Warwick Symposium on Operator Algebras and Applications, organized by David Evans and supported by S.E.R.C. I am also grateful to Charles Batty for several stimulating discussions in this area.

\section{The generalization of Glimm's result}

Let $A$ be a $C^{*}$-algebra and suppose that pure states $\phi$ and $\psi$ have support projections $p$ and $q$ in $A^{* *}$. As in $[4,12]$, we define the transition probability $\langle\phi, \psi\rangle$ between $\phi$ and $\psi$ by

$$
\langle\phi, \psi\rangle=\phi(q)=\psi(p) .
$$

If $\phi$ and $\psi$ are inequivalent then $\langle\phi, \psi\rangle=0$. On the other hand, if $\phi$ and $\psi$ are equivalent there exists an irreducible representation $\pi$ of $A$ and unit vectors $\xi$ and $\eta$ in the Hilbert space for $\pi$ such that

$$
\phi(a)=\langle\pi(a) \xi, \xi\rangle \text { and } \psi(a)=\langle\pi(a) \eta, \eta\rangle
$$

for all $a \in A$. In this case $\langle\phi, \psi\rangle=|\langle\xi, \eta\rangle|^{2}$.

The following result is noted in [4, Remark 2] as a simple consequence of the equality

$$
\|\phi-\psi\|=2(1-\langle\phi, \psi\rangle)^{1 / 2}
$$

(see [8, Corollary 9], $[9$, p. 146] and [11, Lemma 2.4]).

Lemma 1. Let $A$ be a $C^{*}$-algebra, let $T: P(A) \times P(A) \rightarrow[0,1]$ be defined by

$$
T(\phi, \psi)=\langle\phi, \psi\rangle \quad((\phi, \psi) \in P(A) \times P(A)),
$$

and let $P(A) \times P(A)$ be endowed with the product weak ${ }^{*}$-topology. Then $T$ is continuous at $(\phi, \psi)$ whenever $\langle\phi, \psi\rangle=0$.

The next lemma is the key to the generalization of Glimm's result.

Lemma 2. Let $\phi$ and $\psi$ be inequivalent pure states of a $C^{*}$-algebra $A$. Suppose that there exists a net $\left(\pi_{\alpha}\right)$ of irreducible representations of $A$ and, for each $\alpha$, unit vectors $\xi_{a}$ and $\eta_{\alpha}$ such that

$$
\left\langle\pi_{\alpha}(\cdot) \xi_{\alpha}, \xi_{\alpha}\right\rangle \rightarrow \phi \text { and }\left\langle\pi_{\alpha}(\cdot) \eta_{\alpha}, \eta_{\alpha}\right\rangle \rightarrow \psi
$$


Then $\left\langle\xi_{\alpha}, \eta_{\alpha}\right\rangle \rightarrow 0$ and $\left\langle\pi_{\alpha}(a) \xi_{\alpha}, \eta_{\alpha}\right\rangle \rightarrow 0$ for all $a \in A$.

Proof. Let $\tilde{A}$ be the $C^{*}$-algebra generated by $A$ and 1 in $A^{* *}$ and let $\tilde{\pi}_{\alpha}$ be the canonical extension of $\pi_{\alpha}$ to $\tilde{A}$. Let $u$ be a unitary element of $\tilde{A}$. By linearity, it suffices to show that $\left\langle\tilde{\pi}_{\alpha}(u) \xi_{\alpha}, \eta_{\alpha}\right\rangle \rightarrow 0$.

Writing $v_{\alpha}=\tilde{\pi}_{\alpha}(u)$, we have

$$
\left\langle\pi_{\alpha}(a) v_{\alpha} \xi_{\alpha}, v_{z} \xi_{\alpha}\right\rangle \rightarrow \phi\left(u^{*} a u\right)
$$

for all $a \in A$. Since $\psi$ and $\phi\left(u^{*} \cdot u\right)$ are inequivalent we have $\left\langle\phi\left(u^{*} \cdot u\right), \psi\right\rangle=0$ and hence by Lemma 1

$$
\left|\left\langle v_{\alpha} \xi_{\alpha}, \eta_{\alpha}\right\rangle\right|^{2} \rightarrow 0
$$

Glimm's proof of the above lemma in the liminal case uses functional calculus to convert a compact operator into one of finite rank. This leads to an element of $A$ which exhibits the difference between $\operatorname{ker} \pi_{\phi}$ and $\operatorname{ker} \pi_{\psi}$. In general, however, these kernels might coincide. Nevertheless, it is in fact possible to modify Glimm's argument as follows (although, for conceptual reasons, we prefer the proof given above). Let $\xi$ and $\eta$ be the GNS vectors for $\phi$ and $\psi$ respectively. Given $b \in A$ there exists, by Kadison's transitivity theorem, $a=a^{*} \in A$ such that $\pi_{\psi}(a) \pi_{\psi}(b) \eta=0$ and $\pi_{\phi}(a) \xi=\xi$. By functional calculus we may assume $0 \leqq a \leqq 1$. Then we may use Glimm's calculations in [7, pp. 603604] to show that $\left\langle\pi_{\alpha}(b) \eta_{\alpha}, \xi_{\alpha}\right\rangle \rightarrow 0$ and $\left\langle\xi_{\alpha}, \eta_{\alpha}\right\rangle \rightarrow 0$.

Theorem 1. Let $A$ be a $C^{*}$-algebra and let $\phi_{1}, \phi_{2}, \ldots, \phi_{n}$ be pure states of $A$ which are pairwise inequivalent. The following conditions are equivalent.

(i) There exist positive real numbers $\lambda_{1}, \lambda_{2}, \ldots, \lambda_{n}$ with unit sum such that

$$
\sum_{i=1}^{n} \lambda_{i} \phi_{i} \in \overline{P(A)}
$$

(ii) There exists a net $\left(\pi_{\alpha}\right)$ in $\hat{A}$ such that $\pi_{\alpha} \rightarrow \pi_{\phi_{i}}$ for each $i(1 \leqq i \leqq n)$.

(iii) Whenever $\mu_{1}, \ldots, \mu_{n}$ are non-negative real numbers with unit sum,

$$
\sum_{i=1}^{n} \mu_{i} \phi_{i} \in \overline{P(A)}
$$

Proof. (i) $\Rightarrow$ (ii). Let $\phi=\sum_{i=1}^{n} \lambda_{i} \phi_{i}$ and let $\left(\phi_{a}\right)$ be a net in $P(A)$ such that $\phi_{\alpha} \rightarrow \phi$. Fix $i \in\{1,2, \ldots, n\}$ and let $J$ be an ideal of $A$ such that $\pi_{\phi_{i}}(J) \neq\{0\}$. Then $\phi_{i}(J) \neq\{0\}[6,2.4 .9]$ and hence $\phi\left(J \neq\{0\}\right.$. Thus there exists $\alpha_{0}$ such that $\phi_{\alpha}(J) \neq\{0\}$ for all $\alpha \geqq \alpha_{0}$. Hence $\pi_{\phi_{a}}(J) \neq\{0\}$ for all $\alpha \geqq \alpha_{0}$. This shows that $\pi_{\phi_{z}} \rightarrow \pi_{\phi_{i}}$ (see [6, Section 3]).

(ii) $\Rightarrow$ (iii). Having Lemma 2 at our disposal, we can proceed with a generalization of 
Glimm's method as follows. Let $n$ be a base of weak*-open neighbourhoods of zero in $A^{*}$. Let $N \in n$. The canonical image of $\left(\phi_{i}+N\right) \cap P(A)$ in $\hat{A}$ is an open neighbourhood $V_{i}$ of $\pi_{\phi_{i}}(1 \leqq i \leqq n)[6,3.4 .11]$. By (ii), there exists an irreducible representation $\pi_{N}$ of $A$ such that $\pi_{N} \in V_{i}$ for all $i(1 \leqq i \leqq n)$. Hence there exist unit vectors $\xi_{1}^{(N)}, \xi_{2}^{(N)}, \ldots, \xi_{n}^{(N)}$ in the Hilbert space for $\pi_{N}$ such that

$$
\phi_{i}^{(N)} \in \phi_{i}+N(1 \leqq i \leqq n),
$$

where

$$
\phi_{i}^{(N)}(a)=\left\langle\pi_{N}(a) \xi_{i}^{(N)}, \xi_{i}^{(N)}\right\rangle(a \in A) .
$$

For each $i$, the net $\left(\phi_{i}^{(N)}\right)$, indexed by $n$ in the obvious way, is convergent to $\phi_{i}$. It follows from Lemma 2 that if $i \neq j$ and $a \in A$ then

$$
\left\langle\xi_{i}^{(N)}, \xi_{j}^{(N)}\right\rangle \rightarrow 0 \text { and }\left\langle\pi_{N}(a) \xi_{i}^{(N)}, \xi_{j}^{(N)}\right\rangle \rightarrow 0 .
$$

Let $\xi^{(N)}=\sum_{i=1}^{n} \mu_{i}^{1 / 2} \xi_{i}^{(N)}$. By (1), $\left\|\xi^{(N)}\right\| \rightarrow 1$. Eventually $\left\|\xi^{(N)}\right\| \neq 0$ and then we may form $\eta^{(N)}=\left\|\xi^{(N)}\right\|^{-1} \xi^{(N)}$. We define $\psi_{N} \in P(A)$ by

$$
\psi_{N}(a)=\left\langle\pi_{N}(a) \eta^{(N)}, \eta^{(N)}\right\rangle(a \in A)
$$

Using (1) again, it is routine to check that

$$
\psi_{N} \rightarrow \sum_{i=1}^{n} \mu_{i} \phi_{i}
$$

(iii) $\Rightarrow$ (i). This is immediate.

The continuity of the canonical map from $P(A)$ to $\hat{A}$ ensures that the above net $\left(\pi_{N}\right)$ converges to $\pi_{\phi_{i}}$ for each $i$. However, $\left(\pi_{N}\right)$ might not be a subnet of $\left(\pi_{\alpha}\right)$. If in other circumstances a subnet is required, one may index with pairs $(N, \alpha)$, choosing $\pi_{N, \alpha}$ to be some $\pi_{\beta}$ where $\beta \geqq \alpha$ and $\pi_{\beta} \in V_{i}$ for all $i$.

The fact that (i) implies (ii) in Theorem 1 is also valid without the assumption that the $\phi_{i}$ 's are pairwise inequivalent.

Corollary. Let $\phi$ and $\psi$ be inequivalent pure states of a $C^{*}$-algebra A. The following conditions are equivalent.

(i) $\frac{1}{2}(\phi+\psi) \in \overline{P(A)}$.

(ii) $\pi_{\phi}$ and $\pi_{\psi}$ cannot be separated by disjoint open subsets of $\hat{A}$.

We note that the above Corollary closes gaps in the proofs of [3, Theorem 2.8, (4) $\Rightarrow(5)]$ and $[5$, Theorem 3.5 , (iii) $\Rightarrow$ (i)] which arose from overlooking the role of liminality in the construction in [7].

The next result is a version of Theorem 1 for infinite convex combinations. 
Theorem 2. Let $A$ be a $C^{*}$-algebra and let $\left(\phi_{i}\right)_{i \geqq 1}$ be a sequence of pairwise inequivalent pure states of $A$. The following conditions are equivalent.

(i) There exists a sequence $\left(\lambda_{i}\right)_{i \geqq 1}$ of positive real numbers such that $\sum_{i=1}^{\infty} \lambda_{i}=1$ and the norm-convergent sum $\sum_{i=1}^{\infty} \lambda_{i} \phi_{i}$ lies in $\overline{P(A)}$.

(ii) There exists a net $\left(\pi_{\alpha}\right)$ in $\hat{A}$ such that $\pi_{\alpha} \rightarrow \pi_{\phi_{i}}$ for all $i$.

(iii) Whenever $\left(\mu_{i}\right)_{i \geq 1}$ is a sequence of non-negative real numbers such that $\sum_{i=1}^{\infty} \mu_{i}=1$, $\sum_{i=1}^{\infty} \mu_{i} \phi_{i} \in \bar{P}(A)$.

Proof. (i) $\Rightarrow$ (iii). This is proved as in Theorem 1. (ii) $\Rightarrow$ (iii). By truncating and scaling, we see that $\sum_{i=1}^{\infty} \mu_{i} \phi_{i}$ is the norm-limit of a sequence of finite convex combinations of the $\phi_{i}$ 's, each of which lies in $\overline{P(A)}$ by Theorem 1 . Since $\overline{P(A)}$ is norm-closed, it contains $\sum_{i=1}^{\infty} \mu_{i} \phi_{i}$. (iii) $\Rightarrow$ (i). This is immediate.

\section{An application}

Theorem 3. Let $J$ be a proper primal ideal of a separable $C^{*}$-algebra $A$. Then there exists $\phi \in \overline{P(A)} \cap S(A)$ such that $J=\operatorname{ker} \pi_{\phi}$.

Proof. Since $A / J$ is separable, its primitive ideal space is separable [10, 4.3.4]. Thus there is a countable set $S$ of distinct primitive ideals of $A$ with intersection equal to $J$. Since $J$ is primal, it follows from [2, Proposition 3.2] that there exists a net $\left(P_{a}\right)$ of primitive ideals of $A$ such that $P_{\alpha} \rightarrow P$ for all $P \in S$.

Suppose that $S$ is infinite, say $S=\left\{P_{1}, P_{2}, \ldots,\right\}$. Let $\phi_{i}$ be a pure state of $A$ such that $P_{i}=\operatorname{ker} \pi_{\phi_{i}}$ for each $i$. Since $P_{i} \neq P_{j}, \phi_{i}$ and $\phi_{j}$ are inequivalent for $i \neq j$. Let $\pi_{\alpha}$ be an irreducible representation of $A$ such that $P_{\alpha}=\operatorname{ker} \pi_{\alpha}$ for each $\alpha$. Then $\pi_{\alpha} \rightarrow \pi_{\phi_{i}}$ for each $i$ and so

$$
\phi=\sum_{i=1}^{\infty} 2^{-i} \phi_{i} \in \overline{P(A)}
$$

by Theorem 2. Moreover,

$$
\operatorname{ker} \pi_{\phi}=\bigcap_{i=1}^{\infty} P_{i}=J
$$

On the other hand, suppose that $S$ is finite, say $S=\left\{Q_{1}, Q_{2}, \ldots, Q_{n}\right\}$ for some $n \geqq 1$. Taking $\psi=(1 / n) \sum_{i=1}^{n} \psi_{i}$, where $\psi_{i} \in P(A)$ and $\operatorname{ker} \pi_{\psi_{i}}=Q_{i}$, we have that $\operatorname{ker} \pi_{\psi}=J$ and $\psi \in \overline{P(A)}$ by Theorem 1 .

\section{REFERENCES}

1. R. J. ArChBoLD, Topologies for primal ideals, J. London Math. Soc. (2) 36 (1987), 524-542. 
2. R. J. ArChbold and C. J. K. Batty, On factorial states of operator algebras, III, J. Operator Theory 15 (1986), 53-81.

3. R. J. Archbold and C. J. K. Batty, Homogeneous states of $C^{*}$-algebras, Quart. J. Math. Oxford (2) 38 (1987), 259-275.

4. R. J. Archrold and F. W. Shultz, Characterization of $C^{*}$-algebras with continuous trace by properties of their pure states, Pacific J. Math., to appear.

5. C. J. K. Battr and R. J. ARChBold, On factorial states of operator algebras, II, J. Operator Theory 13 (1985), 131-142.

6. J. Dixmier, Les $C^{*}$-algèbres et leurs représentations, 2nd edition (Gauthier-Villars, Paris, 1969).

7. J. Glıмm, Type I $C^{*}$ algebras, Ann. of Math. 73 (1961), 572-612.

8. J. Glimm and R. V. Kadison, Unitary operators in $C^{*}$-algebras, Pacific J. Math. 10 (1960), 547-556.

9. R. V. Kadison, Limits of states, Comm. Math. Phys. 85 (1982), 143-154.

10. G. K. Pedersen, $C^{*}$-algebras and their automorphism groups (Academic Press, London, 1979).

11. R. T. Powers and E. Størmer, Free states of the canonical anticommutation relations, Comm. Math. Phys. 16 (1970), 1-33.

$12^{\bullet}$ F. W. Shultz, Pure states as a dual object for $C^{*}$-algebras, Comm. Math. Phys. 82 (1982), 497-509.

Department of Mathematics

The EdWARd Wright BuILDING

Dunbar Street

Aberdeen AB9 2TY 